\title{
Progress in VLBI Stellar Astrometry for the NASA/Stanford Relativity Mission (Gravity Probe B)
}

\author{
M. I. Ratner, D. E. Lebach, \& I. I. Shapiro
}

Harvard-Smithsonian Center for Astrophysics, Cambridge, MA 02138, USA

\author{
N. Bartel, M. F. Bietenholz, \& R. R. Ransom \\ Dept. of Physics and Astronomy, York University, North York, ON M3J 1P3, \\ Canada
}

J.-F. Lestrade

Observatoire de Meudon, F-92195 Meudon, Principal Cedex, France

\begin{abstract}
The NASA/Stanford Relativity Mission (Gravity Probe B) is to test the unverified "frame-dragging" prediction of general relativity through measurements of the precessions of orbiting gyroscopes. For mission accuracy goals to be met, the proper motion of a "guide star," whose position will be used as an inertial reference, must be determined in an extragalactic reference frame with a standard error less than 0.5 mas $/ y r$. We discuss our VLBI observations of the current guide-star candidates (radio stars HR 1099, HR 5110, and HR 8703) and our techniques for obtaining differential astrometric positions with the needed accuracy.
\end{abstract}

\section{Role of Astrometric VLBI}

The NASA/Stanford Relativity Mission (Gravity Probe B, or GP-B) will monitor the precessions of four high-precision gyroscopes in a single spacecraft scheduled to be launched into a polar orbit some time between December 1999 and October 2000. The main goal of the mission is to measure the "frame-dragging" effect due to the diurnal rotation of the earth's mass (Turneaure, Everitt, \& Parkinson 1986) with a standard error of 0.5 mas/yr or less. The precessions of the gyroscopes will be measured relative to the apparent position of one bright star used as a "guide star." Because errors in the estimate of the proper motion of the guide star map directly into errors in the measured precessions, the proper motion of the guide star is also needed at this level of accuracy, and with a sufficiently high degree of confidence that the GP-B mission usefully tests general relativity and not just the astrometry. We have identified adequately bright and suitably located stars that are also radio stars for which proper motions can be determined in the extragalactic frame with VLBI (Ratner et al. 1996; Ransom et al. $1996 \mathrm{a}, \mathrm{b})$. VLBI is the astrometric technique of choice, not only because it has already proven capable of yielding the required accuracy (e.g., Bartel et al. 1986; Lestrade et al. 1995), but also because the cores of extragalactic radio sources are appropriate position references against which to measure the rotation of local inertial frames.

\section{Observations and Analysis}

Each of the three best guide-star candidates, namely the RS CVn radio binary stars HR 1099 (V711 Tau), HR5110 (BH CVn), and HR 8703 (IM Peg), have already been observed with astrometric VLBI at several earlier epochs between 1987 and 1993 as part of a program by one of us (Lestrade et al. 1995) to tie the 
Hipparcos results to the extragalactic reference frame. From those observations, proper motions with statistical standard errors (in both coordinates) of 0.31 , 0.13 , and $0.47 \mathrm{mas} / \mathrm{yr}$, respectively, have been obtained. However, the crucial role played in the GP-B mission by its one guide star requires that we estimate, in addition to the usual astrometric parameters, a constant proper acceleration to model the possible effects of any relatively distant companion of the radio binary (such as an unseen $\mathrm{M}$ dwarf). To minimize the number of additional observations required, we are trying to improve upon the accuracy of each individual position determination, in part by using additional reference sources.

A program of about four additional VLBI epochs per year, extending through the year of the mission, will adequately determine the rate of motion on the sky of any one of these candidates, with explicit estimation of a constant acceleration, if a position with standard error 0.5 mas or less can be determined at each observing epoch, and if the random, i.e., non-orbital motion of the stellar radio source also does not exceed this amount. For HR 5110, but not the other two stars, there are already enough high-quality position determinations to acceptably bound this random motion. To check on the other two, within the past year (May 1996-Jan 1997) we made astrometric VLBI observations, not yet fully reduced, at two epochs for each star scheduled so that the orbital phases of the two observations were nearly equal. By using the VLBA together with two of NASA's $70 \mathrm{~m}$ dishes and other sensitive antennas, we obtained sufficient sensitivity at $3.6 \mathrm{~cm}$ to be nearly assured of detecting the radio stars in only $\sim 3 \mathrm{~min}$ of integration on at least several baselines. Such detections allow for mapping (phase-referenced and otherwise) and for astrometry by least-squares analysis of phase delays (e.g., Bartel et al. 1986). We will thus be able to compare the astrometric results obtained from least-squares analysis with those obtained from the independent phase-referenced mapping approach (e.g., Lestrade et al. 1990).

In both approaches, we must account for the effects of source structure. Since the stellar flux density during some of our observation sessions varied by up to 200 percent over a few hours, we must develop techniques for mapping sources with rapidly changing flux density. Only then can we adequately investigate the possibility of significant non-orbital source motions within a given session.

Acknowledgments. This research has made use of the Simbad database, operated at CDS, Strasbourg, France, and the facilities of the National Radio Astronomy Observatory, funded by the National Science Foundation, and operated under a cooperative agreement by Associated Universities, Inc.

\section{References}

Bartel, N, et al. 1986. Nature, 319, 733-738.

Lestrade, J.-F, et al. 1990. $A J, \mathbf{9 9}, \mathbf{1 6 6 3 - 1 6 7 3 .}$

Lestrade, J.-F. et al. 1995. A\&A, 304, 182-188.

Ransom, R. R. et al. 1996a. BAAS, 28, 1418.

Ransom, R. R. et al. 1996b. J. Roy. Astron. Soc. Canada, 90, 318.

Ratner, M. I., et al. 1996. in Proceedings of the Seventh Marcel Grossmann Meeting on General Relativity, eds. R. T. Jantzen \& G. M. Keiser (Singapore: World Scientific), p. 1553-1554.

Turneaure, J. P., Everitt, C. W. F., \& Parkinson, B. W. 1986. in Proceedings of the Fourth Marcel Grossmann Meeting on General Relativity, ed. R. Ruffini (Amsterdam: NorthHolland), p. 411-463. 